\title{
Generation of an UWB monocycle employing cross- phase modulation in a SOA-MZ Interferometer
}

\author{
Manuel Rius, José Mora, José Capmany \\ ITEAM Research Institute, Universitat Politécnica de \\ Valencia \\ Valencia, Spain \\ jmalmer@upv.es
}

\author{
Vanessa Moreno, Miguel A. Muriel \\ Department of Photonics Technology and \\ Bioengineering, Universidad Politécnica de Madrid \\ Madrid, Spain \\ m.muriel@upm.es
}

\begin{abstract}
In the present article, an innovative approach for generation of an UWB monocycle is proposed and experimentally demonstrated. The proposed design features the combination of an interferometric device (SOA-Mach Zehnder interferometer) with an optical processor unit. The fusion of such components permits to generate, combine and customize UWB pulses. An optical pulse is used as pump signal and two optical carriers represent and the optical input of the system. The selection of a specific wavelength and therefore of a particular port provides the possibility of modifying the systems output pulse polarity. The capacity of transmitting several data sequence has been also evidenced.
\end{abstract}

Keywords-optical pulse generation; ultrawideband technology; interferometric structure; non-linear effects, reconfigurability

\section{INTRODUCTION}

Ultra-wideband (UWB) technology represents one of the most interesting techniques for short range, high data rate and capacity communications wireless systems due to its valuable advantages, such as immunity to multipath fading, being carrier free, broad bandwidth and low power spectral density [1]. The US Federal Communications Commission (FCC) defines UWB as any signal that occupies more than $500 \mathrm{MHz}$ bandwidth or possesses a fractional bandwidth greater than $20 \%$. In this context, the FCC has also approved the unlicensed use of a spectral band from 3.1 to $10.6 \mathrm{GHz}$ with a transmitted power spectral density (PSD) lower than -41.3 $\mathrm{dBm} / \mathrm{MHz}$ for indoor wireless communications [2].

Parallel to the increasing use of UWB technology, there has been a considerable interest in the implementation of photonic solutions in order to profit the benefits of Microwave Photonics (MWP) [3]. For instance, the range of distance of an UWB scheme is limited to a few meters to tens of meters. To enlarge the area of coverage, MWP leads to bring an elegant solution through the distribution of the UWB signals in the optical domain or UWB over fiber (UWBoF) [4]. The inherent advantages of the optical domain such as low losses, lightweight, high bandwidth, tunability, reconfigurability and immunity to electromagnetic interference are useful in the process of the UBW signal generation. Moreover, it is highly desirable that the UWB signals can be generated directly in the optical domain without the need of electrical-optical conversion. However, MWP is not only employed to UWB signals distribution but also for UWB signal generation, processing and control.

Both Gaussian monocycle and doublet pulses which are first- and second-order derivatives of Gaussian pulse respectively are considered as promising candidates for UWB communications. The Gaussian monocycle pulse possesses lower bit error rate, better multipath performance and wider bandwidth compared with other impulse signals [5]. Furthermore, the doublet or Scholtz's monocycle pulse has less energy in low frequency than the mentioned monocycle pulse therefore. It holds better performance in UWB systems. Additionally, it can be used in geolocation and positioning applications because of the longer total bi-pulse width [6].

Techniques related to the generation of UWB pulses by means of SOAs represent a field with an extensive background. The employment of these devices has become a valid alternative for all-optical generation due to its nonlinear effects, low power consumption, flexibility and scalability. These implementations can be classified according to the nonlinearity taken into consideration and created waveform. In [7], the nonlinear response of the transfer function can be achieved for currents far from maximum operation points based on the nonlinear transfer function of an EOM. In [8], a monocycle is obtained through a filter-free pump-probe scheme that exploits the cross-gain modulation (XGM) effect of the SOA. Another example of the application of nonlinearities is represented by [9] in which the crosspolarization modulation (XPM) is employed to generate different types of doublets and monocycle. Generation and data transmission are also plausible and demonstrated in [10], in which a pair of polarity-reversed UWB monocycle pulses are generated and Bi-Phase modulation of such waveform is achieved by applying nonreturn-to-zero (NRZ) data to the radio frequency (RF) port of a Mach-Zehnder Modulator.

In this paper, a novel scheme for generation of an UWB monocycle is proposed and experimentally demonstrated. The suggested design exploits the properties of a Mach-Zehnder interferometer (MZI) that is composed of two semiconductor optical amplifier (SOAs), one per each branch, along with the reconfigurability capabilities of an optical processor unit. Two optical pulsed signals are employed as probe wave signals in order to induce cross-phase modulation (XPM) in the SOA- 
MZI. By introducing the previously mentioned inputs through a specific wavelength and therefore by a particular port, it is feasible to modify the systems output pulse polarity. The fusion of a positive tap pulse monocycle and a negative one will produce the desired monocycle. Additionally to the generation process, Bi-Phase modulation of a monocycle pulse is also implemented by only adjusting metrics of the pump signal.

\section{PRINCIPLE OF OPERATION}

Figure 1 exhibits the proposed scheme for generation of an UWB monocycle. It corresponds to a microwave photonics filter in which the key component is a SOA-MZI, that contains two $1 \mathrm{~mm}$ SOAs InGaAsP for low polarizations sensitivity, such device has been previously analyzed in [11].The design contemplates a pump signal centered at an optical wavelength (labeled as $\lambda_{\text {PUMP }}$ ) of $1535.04 \mathrm{~nm}$. The inputs are represented by two continuous optical signals (tagged as $\lambda_{1}$ and $\lambda_{2}$ ) that are injected to the interferometric structure by different ports, $\mathrm{P}_{4}$ and $\mathrm{P}_{5}$, respectively. The selection of one port or another will determine the polarity of the obtained pulse. The monocycle is a product of the combination of the positive and negative tap pulse generated in this arrangement.

The reconfiguration feature is supplied by the optical processing unit. An assemblage of variable delay lines (VDLs) and attenuators, in order to customize the achieved monocycle in terms of delay and amplitude. The systems outcome will be released through port $\mathrm{P}_{2}$ and detected by means of a photodetector (PD).

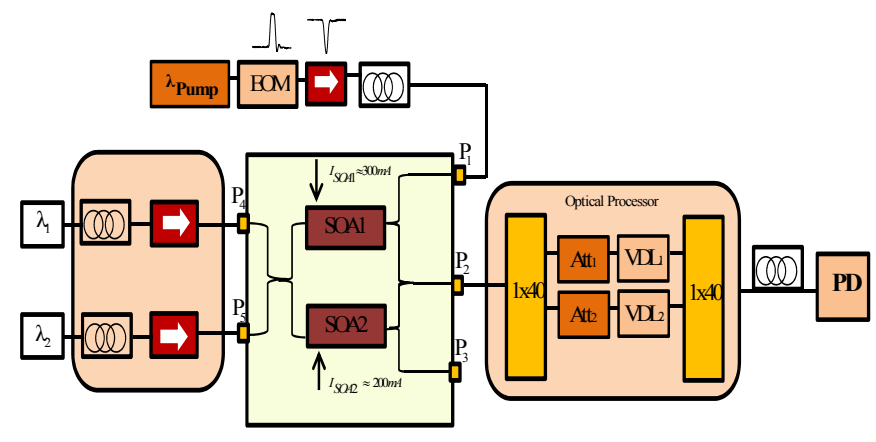

Fig.1 Experimental layout for UWB signal generation.

In principle, cross-gain modulation (XGM) and cross-phase modulation (XPM) processes could be present in the SOAs. However, the linewidth enhancement factor of these devices is large enough to neglect the XGM effect. In this implementation, both SOAs hold a fixed current value, the SOA in the upper branch of the interferometric structure (SOA1) is in possession of a constant current of $300 \mathrm{~mA}$ and the SOA of the lower branch (SOA2) has a current value of $200 \mathrm{~mA}$ approximately.

\section{A. Positive Pulse Generation}

At first, we select the optical wavelength for $\lambda_{1}$ at 1550.12 $\mathrm{nm}$. The bit sequence corresponding to the pump signal is set with a fixed pattern of one " 1 " and sixty-three "0", summing up a total of 64 bits, at a repetition rate of $12.5 \mathrm{GHz}$. The optical power related to this source is $5.29 \mathrm{dBm}$. The obtained waveform after the modulation stage (EOM) corresponds to a Gaussian pulse with a switch of polarization when compared to the pump signal. Both the input pulse and the modulated optical carrier are represented in Figure 2(a) and Figure 2(b).
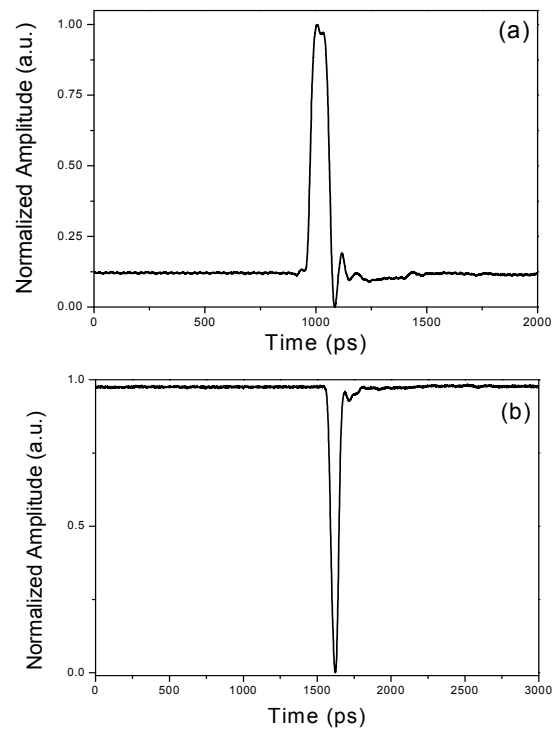

Fig.2 a) Electrical input pulsed signal and (b) modulated optical pulse which is employed as pump signal

If the modulated pulse is launched into the interferometry unit through the port P4, the SOA-MZI generates a pulse with positive tap. Parameters as the amplitude, polarity and time delay can be controlled without any additional step in the experimental design. Figure 3(a) and 3(b) display the positive tap pulse obtained and the corresponding spectrum respectively.
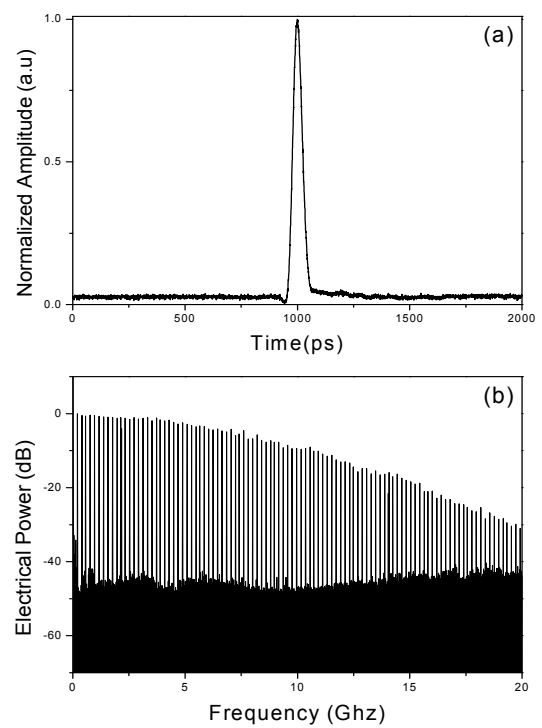

Fig.3 a) Positive tap pulse generated and (b) corresponding electrical spectrum 


\section{B. Negative Pulse Generation}

Since the architecture submitted employs a counter propagation configuration, no optical filter is needed to separate the pump signal from $-\lambda_{1}$ or $\lambda_{2}$. As it was previously mentioned, the sign of the tap is established by the operation current point to perform the wavelength conversion. Therefore it is possible to achieve a positive or negative coefficient by selecting the adequate input port, in this case port $\mathrm{P}_{5}$ (which is related to $\lambda_{2}$ ) will provide with a negative coefficient pulse.

For this implementation, we settle an optical wavelength for $\lambda_{2}$ of $1552.52 \mathrm{~nm}$. The bit sequence of the pump signal matches with the one employed in the generation process of a positive tap pulse. The optical power value employed in this process is $4.44 \mathrm{dBm}$. In this case, the polarity of the accomplished pulse matches with the modulated pump signal. Figure 4(a) plots the negative tap pulse achieved and Figure 4(b) shows the spectrum related to such waveform.
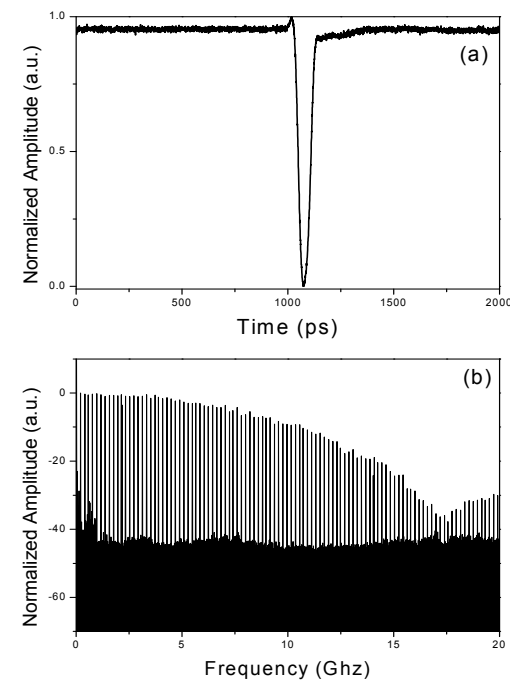

Fig.4 a) Negative tap pulse generated and (b) corresponding electrical spectrum.

\section{EXPERIMENTAL RESULTS}

Considering that the achieved monocycle is a result of the combination of positive and negative tap pulses, the configuration of the obtained waveform was monitored by means of an electrical spectrum analyzer (ESA) after the detection through a photodetector (PD).

The referenced configuration process is performed by the optical processing unit, a conglomerate of components that permits to customize the features of amplitude and delay. In this case, once both optical lasers sources $\left(\lambda_{1}\right.$ and $\left.\lambda_{2}\right)$ are activated and their corresponding outcomes visualized, we start modifying the delay between the generated pulses in intervals of 200 ps. We achieve a good match between the waveform generated and the desired monocycle, the configuration procedure concludes. Figure 5(a) depicts the positive and negative pulses generated at their original positions along with the corresponding spectral representation in Figure 5(b). The second position of the incoming scheme along with its spectrum is visualized in Figures 5(c) and 5(d). Finally, the acquired monocycle pulse and it spectrum are illustrated in Figures 5(e) and 5(f).
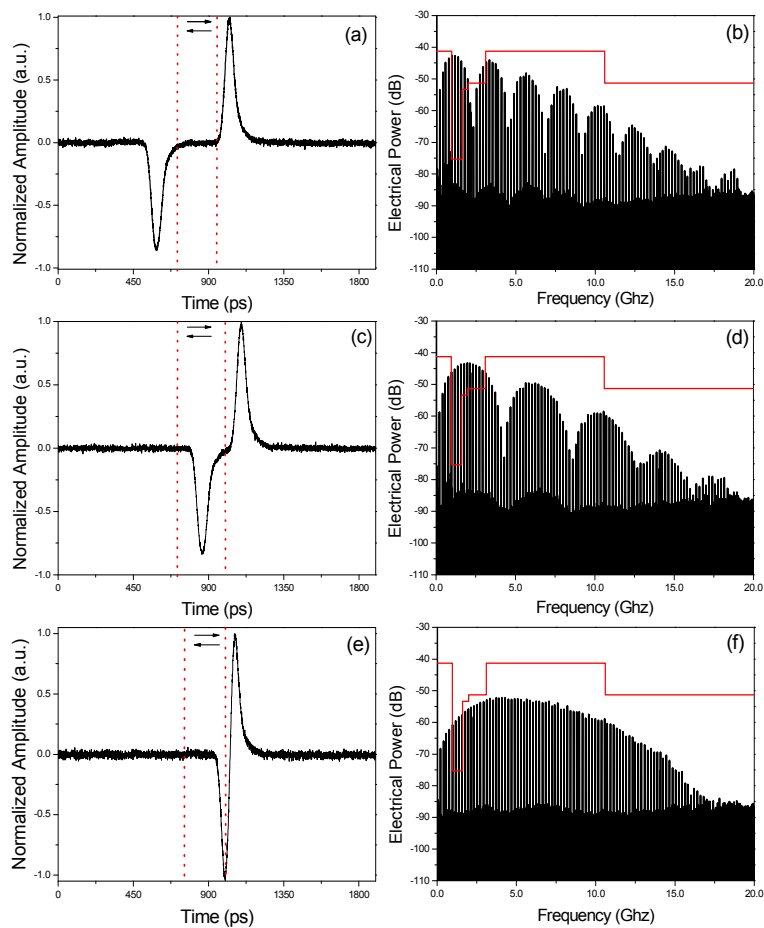

Fig.5 a) Generated pulses in their original position, prior to the delay configuration, b) corresponding spectral representation, c) generated pulses with a 200 picoseconds approximation, d) spectral representation for the modified pulses, e) obtained monocycle waveform after final delay configuration and f) spectrum for the obtained UWB monocycle pulse.

\section{A. Modulation Sequences}

The submitted architecture can be used to perform data transmission through the produced UWB monocycle pulse. As it was analyzed in previous section, the fusion of two complementary positive and negative tap pulses provides an UWB monocycle. By modifying the pump signals, bit sequence it is plausible to appreciate data transmission on the generated signal.

Figure 6 plot three data sequences transmitted and the corresponding spectrums. First data scheme was transmitted with a bit sequence of all ones " 1 " as shown in Figure 6(a). Second data series was composed of both alternated ones " 1 " and zeros " 0 " bits and third data sequence was transmitted randomly as plotted in Figure 6(b) and 6(c), respectively. Meanwhile Figures 6(d), 6(e) and 6(f) depict the corresponding spectrums. 

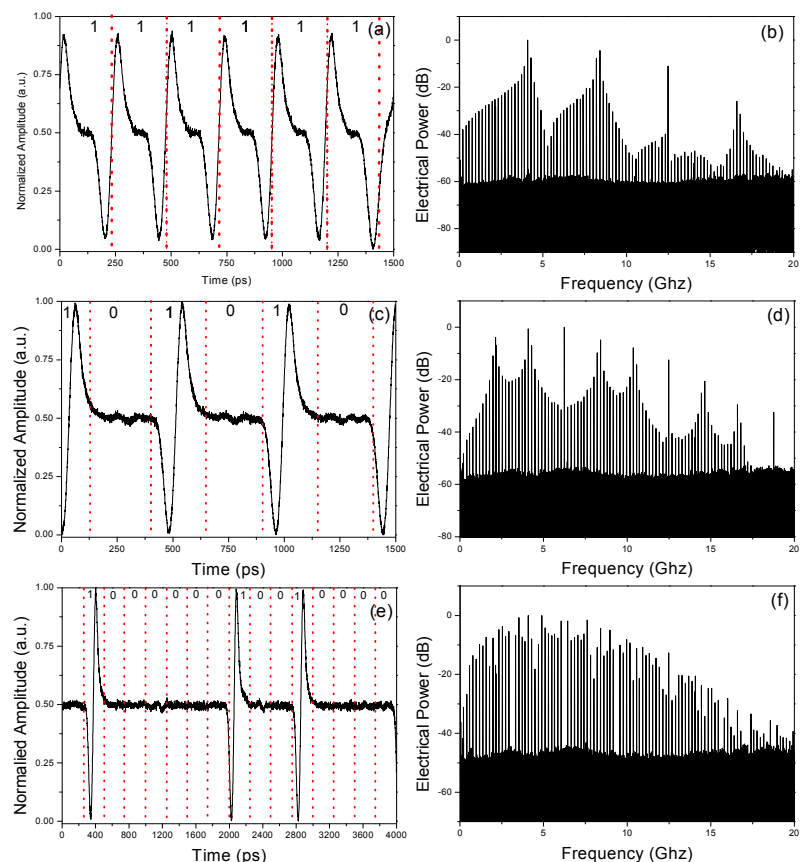

Fig.6 (a) First data scheme transmitted with a bit sequence of all ones "1" and (b) corresponding spectral representation. (c) Second data series composed of both ones " 1 " and zeros " 0 " bits and (d) spectrum related to the second data sequence. (e)Third data sequence transmitted and (f) electrical spectrum for the last data sequence transmitted.

\section{CONCLUSIONS}

A novel approach to generate an UWB monocycle by exploiting the XPM effect present in an interferometric structure has been proposed and experimentally demonstrated. In the proposed setup, the fusion of two pulses with inverted polarities allows to create the desired UWB monocycle pulse. Customization of the generated waveform is performed by means of an optical processor unit. Therefore, reconfiguration and up to a certain point capacity of spectral shaping are valuable characteristics featured by the design. Data transmission was also accomplished through various sequences transmitted.

\section{ACKNOWLEDGMENT}

The research leading to these results has been funded by the national project TEC2010-21303-C04-02 and TEC2011-26642 funded by the Ministerio de Economía y Competitividad and the projects FEDER UPVOV08-3E-008 and UPVOV10-3E492

\section{REFERENCES}

[1] J. Yao, F, Zeng, and Q.Wang, "Photonic generation of ultrawideband signals," IEEE Journal Lightwave, vol. 25, pp. 3219-3235, November 2007.

[2] G. Roberto and G.Rogerson, "Ultra-wideband wireless systems," Microwave Magazine, vol 4, pp. 36-47, June 2003.

[3] J. Capmany and D. Novak, "Microwave photonic combines two worlds," Nature Photon, vol. 1, pp. 319-330, June 2007.

[4] S. Pan and J. Yao, "Performance evaluation of UWB signal transmission over Optical Fiber," IEEE Journal on selected areas in communications, vol. 28, pp. 889-900, August 2010.

[5] X. Chen amd S. Kiaei, "Monocycle shapes for ultrawide-band system," IEEE International Symposium of Circuits and Systems, pp. I597-I600, May 2002.

[6] I. Oppermann, M. Hama la inen and J. Linatti, UWB theory and applications, John Wiley \& Sons, West Sussex, 2004, pp. 3-5

[7] Q. Wang and J. Yao, "UWB doublet generation using nonlinearlybiased electro-optic intensity modulator,"Electronics Letters, vol.42, pp.1304-1305, October 2006.

[8] J. Dong,S. Fu, P. Shum, X Zhang and D. Huang, "Filter free all optical UWB monocycle generation based on the semiconductor optical amplifier (SOA) non linearities," Digest 6th. International Conference on Information, Communications \& Signal Processing Singapore, pp.14, December 2007.

[9] H. Chen, M. Chen, T. Wang, M Li and S. Xie, "Methods for ultrawideband pulse generation based on optical cross polarization modulation," Lightwave Technology Journal, vol 26, pp.2492-2499, August 2008.

[10] Y.Yu, J.Dong, X.Li and X.Zhang, "UWB monocycle generation and biphase modulation based on Mach-Zehnder Modulator and semiconductor optical amplifier," IEEE Photonics Journal, vol. 4, pp.327-339, April 2012.

[11] M.D. Manzanedo, Mora, J. Capmany and J. Mora, "Continuously Tunable Microwave Photonic Filter With Negative Coefficients Using Cross-Phase Modulation in an SOA-MZ Interferometer,'IEEE Photonics Technology Letters, vol. 20, pp. 526-528, April 2008. 